\title{
Hybrid coronary revascularization: the Emory experience
}

\author{
Sorin V. Pusca, Michael E. Halkos \\ Department of Surgery, Division of Cardiothoracic Surgery, Emory University, Atlanta, GA 30322, USA. \\ Correspondence to: Dr. Michael E. Halkos, Division of Cardiothoracic Surgery, Department of Surgery, Emory University, 1364 \\ Clifton Road, Atlanta, GA 30322, USA. E-mail: mhalkos@emory.edu \\ How to cite this article: Pusca SV, Halkos ME. Hybrid coronary revascularization: the Emory experience. Mini-invasive Surg \\ 2021;5:51. https://dx.doi.org/10.20517/2574-1225.2021.45
}

Received: 29 Mar 2021 First Decision: 7 Jun 2021 Revised: 28 Jun 2021 Accepted: 19 Jul 2021 First online: 5 Nov 2021

Academic Editor: Giulio Belli Copy Editor: Xi-Jun Chen Production Editor: Xi-Jun Chen

\begin{abstract}
This article reviews the Emory University Experience with hybrid coronary revascularization and identifies key factors essential for the success of this relatively new and evolving strategy for the treatment of coronary artery disease. Key decisional and technical factors were identified. In addition, careful patient selection, stepwise progression in learning the different aspects of the procedure, and close collaboration between cardiac surgeryinterventional cardiology are key factors for success.
\end{abstract}

Keywords: Hybrid coronary revascularization, robotic coronary bypass, robotic LIMA LAD anastomosis

\section{INTRODUCTION}

Hybrid coronary revascularization (HCR) has evolved over the past decade as a strategy for the treatment of multivessel coronary artery disease combining the most significant advantages of surgical coronary artery bypass (CABG) and coronary artery stenting in order to provide the best possible short- and long-term results with minimal invasion of the patient. The $88 \%-90 \%$ 20-year patency rate of left internal mammary artery (LIMA) to left anterior descending (LAD) coronary artery is the pinnacle of what CABG can offer and is hard to beat ${ }^{[1-4]}$. This excellent patency rate translates into improved survival, improved relief of symptoms, decrease in major adverse cardiac events and decrease the need for reintervention ${ }^{[1-4]}$. Equally important, even though CABG has better long term outcomes than percutaneous coronary intervention (PCI)for multivessel CAD ${ }^{[5]}$, the newer generation drug-eluting stents (DES) deployed to treat discrete 
lesions in non-LAD territories have excellent short term patency rates (6.6\% target vessel failure at 8 months, $8.9 \%$ target vessel failure at 3 years $)^{[6,7]}$ comparable or superior to vein grafts to the same territories (25\% vein graft failure at $12-18$ months $)^{[8]}$ without the morbidity of CABG.

The hybrid strategy has a steep learning curve, particularly on the cardiac surgery side, because the LIMA $\mathrm{LAD}$ anastomosis is done without a sternotomy in an off-pump or beating heart fashion. The strategy also requires seamless teamwork between cardiac surgery and interventional cardiology.

The American College of Cardiology, American Heart Association, American College of Physicians, American Association for Thoracic Surgery, The Preventive Cardiovascular Nurses Association, The Society for Cardiovascular Angiography, and Interventions and The Society of Thoracic Surgeons have issued joint guidelines for HCR, defined as the planned combination of LIMA-to-LAD artery grafting and PCI of one or more non-LAD coronary arteries ${ }^{[9]}$.

Class IIa indications include limitations of traditional CABG (heavily calcified ascending aorta, poor nonLAD target vessels but amenable to PCI, lack of suitable graft conduits) and unfavorable LAD anatomy for PCI (chronic total occlusion or excessive LAD tortuosity).

Class IIb indications include attempts to improve the overall risk-benefit ratio of both PCI and CABG in patients who have multivessel coronary artery disease, would benefit from a LIMA to LAD bypass but have other comorbidities that put them at risk of complications after surgery (recent MI, fraility) or need a rapid return to baseline activities.

This article aims to distill the lessons that we have learned at Emory University over the past decade of application of this strategy and provide guidance on the steps required to introduce it into the armamentarium of an institution.

\section{CABG WITHOUT STERNOTOMY (MINIMALLY INVASIVE CORONARY SURGERY)}

Efforts to avoid partially or completely the sternotomy to perform CABG started in 1995 with Dr. Benetti ${ }^{[10]}$.

At Emory, we moved in the early 2000s to harvest the LIMA using video-assisted thoracic surgery techniques (VATS), a robotic arm, Aesop (Intuitive Surgical, Mountainview, CA) to hold the camera, and a 1.5-2-inch incision, if necessary with resection of costal cartilage, to perform the LIMA to LAD anastomosis off-pump, using a port-based cardiac positioner (Medtronic, Minneapolis, MN. Guidant, Santa Clara, CA, Estech, Danville, CA). The technique was called EndoACAB (endoscopic atraumatic coronary artery bypass). A significant experience of 607 patients was accumulated with excellent results: 30-day mortality of $1 \%$, a mean ICU length of stay of $11.2 \pm 9.9 \mathrm{~h}$, a hospital length of stay of mean $2.4 \pm 1.3$ days, a conversion to sternotomy or standard thoracotomy of $3.6 \%$ (0.7\% emergent) and 5 -year survival of $92.9 \% \pm 2.4 \%{ }^{[11]}$.

Unfortunately, the VATS technique to harvest the mammary was difficult to learn, mainly because the LIMA was harvested with long-shafted instruments endoscopically. Such instruments amplify hand tremors and have no articulation of the distal ends of the instruments to allow complex intrathoracic manipulations. In addition, the Aesop robotic arm, essential for holding the camera, was unfortunately discontinued from production. Furthermore, the advent of the robotic Da Vinci Surgical System (Intuitive Surgical Inc., Sunnyvale, CA) made this procedure somewhat obsolete. 
The Da Vinci robot introduced a new level of precision, visualization, and endoscopic freedom of movement inside the body with 3-dimensional articulating instrumentation and 10x high fidelity magnification. Thus, exposure and visualization were greatly facilitated, and more complex movements could be made inside the chest. Furthermore, the 3-dimensional wristed instruments provided a greater range of motion in a small space. In addition, the robotic instruments are able to filter the hand tremor; hence this becomes a non-issue. Finally, dual consoles allow the technique to be taught much more easily, with seamless transfer of controls between mentor and trainee.

The majority of minimally invasive CABG procedures since 2009 at Emory have been performed with robotic assistance, thus the term robotic-assisted CABG. The procedure has been extensively described in publication ${ }^{[12-16]}$. Briefly, patients are placed supine on the operating table, and after induction, two rolled sheets or a bump is placed under the left should with the superior tip of the bump placed just inferior to the scapula. The patient is positioned slightly towards the left of the table so that when the left arm is loosely tucked, the left shoulder gently hangs off of the bed. The lowering of the left shoulder is critical to avoid conflict with the robotic arms. The patient is then prepped and draped in the usual fashion for coronary bypass surgery; the middle of the chest is marked between the clavicle and costal margin [Figure 1]. There is no specific interspace, but the camera port should be generally placed in the middle of the chest or just slightly lower at approximately the anterior axillary line.

A spinal needle is used to identify the rib and the interspace. We always use a blunt instrument similar to tube thoracostomy prior to inserting the camera port. The camera port can then be placed gently with a slight angle superiorly to avoid underlying cardiac injury. Immediately after this port is placed, carbon dioxide insufflation should be initiated from $8-12 \mathrm{mmHg}$ with careful attention to blood pressure while creating a tension pneumothorax. Not infrequently, anesthesiology will need to make adjustments with loading conditions to allow the patient to tolerate this. The insufflation can be adjusted up or down (6-15 mmHg) as needed. The camera is then inserted, and the superior port is placed 2 interspaces above the camera port in either the 2nd or 3rd interspace, more medial than the camera port.

The inferior port is placed 2-3 interspaces in a similar medial-lateral position as the camera port. Note, the working arm ports should be placed with endoscopic guidance so you can see where the ports are entering the chest and in which interspace [Figure 2]. Finally, the da Vinci system is docked [Figure 3], and the LIMA can be harvested as a thin pedicle or skeletonized.

Our preference is to remove the overlying muscle and fascia to expose the LIMA completely and then take the vessel as a thin pedicle with electrocautery and clips to avoid any manipulation of the artery [Figures 4 and 5]. A small pericardial window posterior to the phrenic is then made, the pericardial fat is dissected off the anterior pericardium, and a full longitudinal pericardiotomy is then made to mirror the same pericardiotomy that would be made via sternotomy [Figure 6].

After dividing the LIMA distally with clips, the robot is undocked, the endoscope and a spinal needle are used to help with localization of the skin incision so that after a $3-4 \mathrm{~cm}$ thoracotomy incision is made, the LAD target should lie directly underneath. No rib retractors are used, but exposure is usually more than adequate with a soft tissue retractor without dividing or resecting any ribs or costal cartilage. The Nuvo stabilizer (Nuvo; Medtronic, Minneapolis, MN) is used to stabilize the LAD; a vessel loop is placed around the more proximal LAD, and tests occluded for 3 min while the LIMA is prepared. 


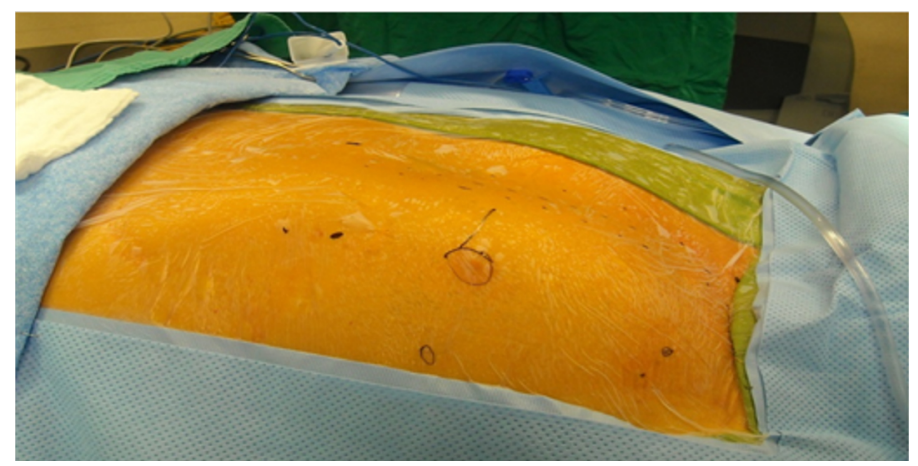

Figure 1. Robotic coronary artery bypass: planning of the incisions.

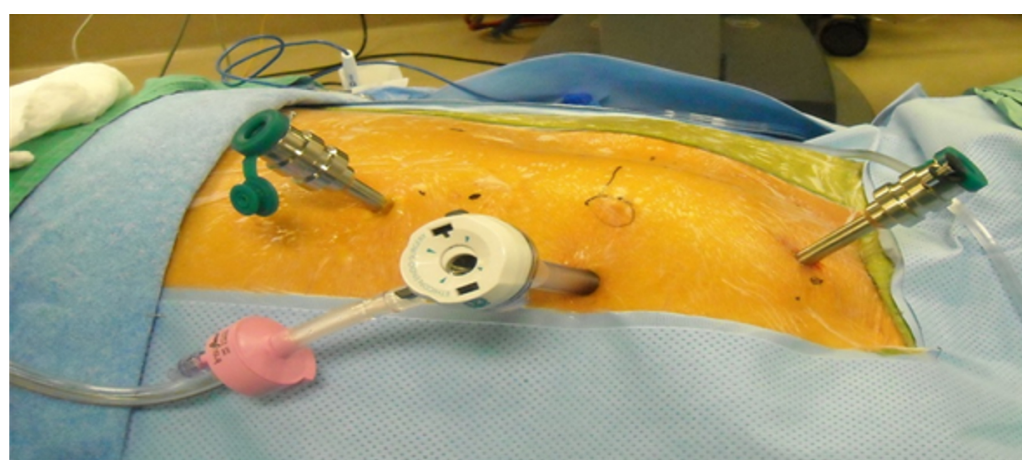

Figure 2. Robotic coronary artery bypass: port insertion.

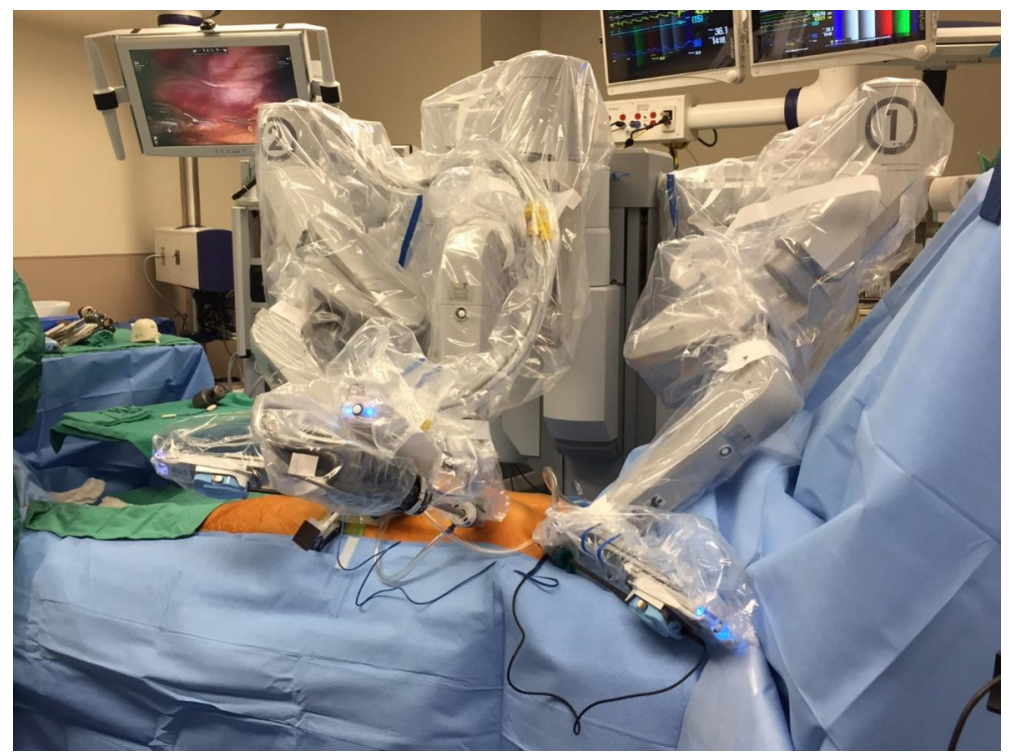

Figure 3. Robotic coronary artery bypass: docking of the Da Vinci robot.

Then the anastomosis is done off-pump with a shunt in place in the same manner that would be done via sternotomy [Figure 7]. 


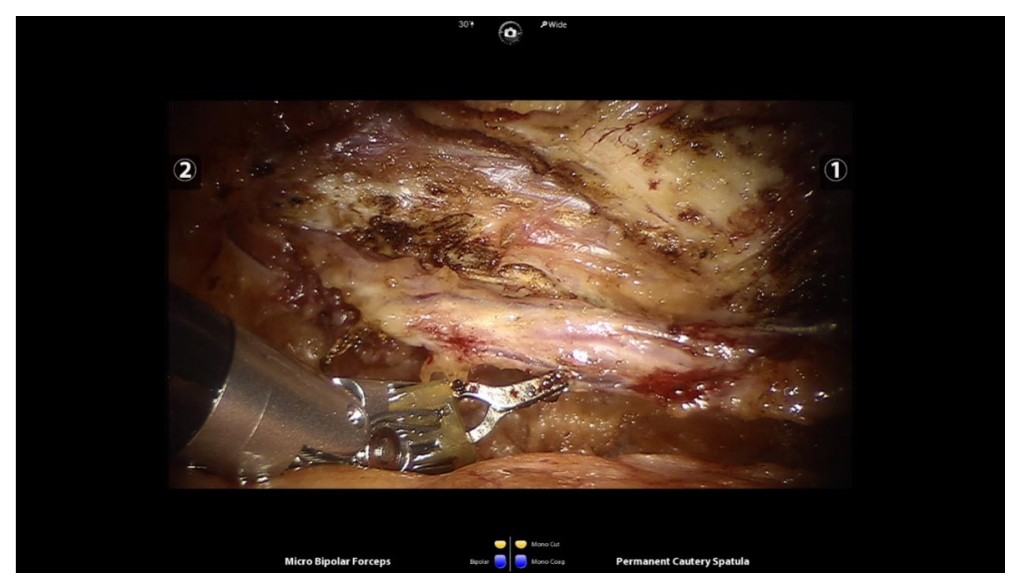

Figure 4. Robotic coronary artery bypass: starting the left internal mammary artery dissection.

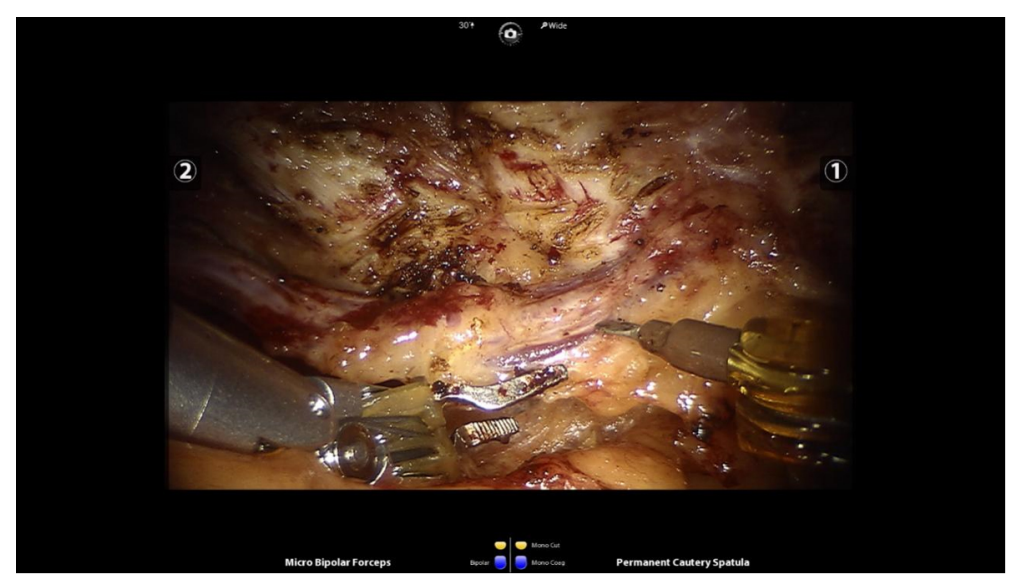

Figure 5. Robotic coronary artery bypass: continuing the left internal mammary artery dissection.

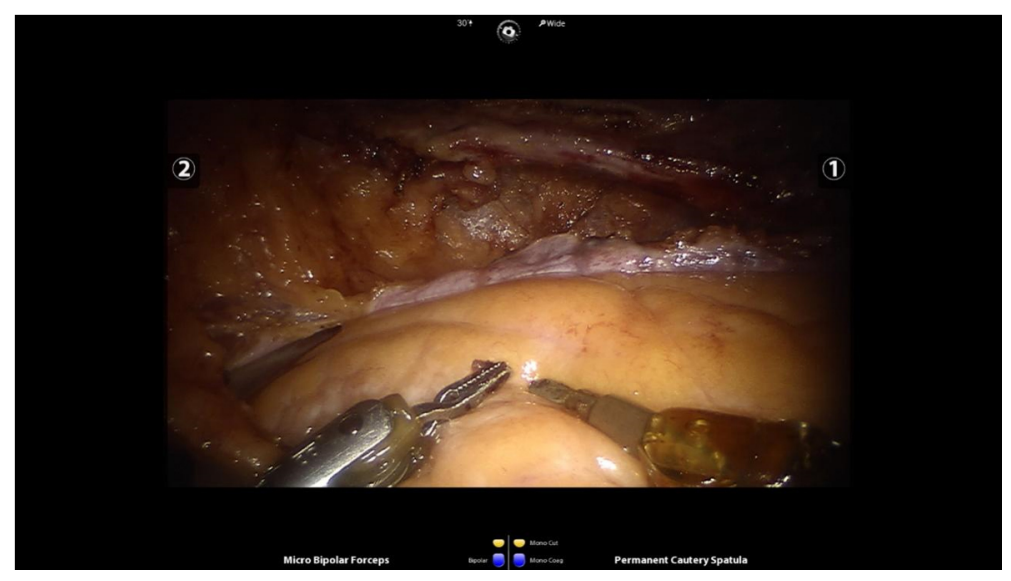

Figure 6. Robotic coronary artery bypass: identifying the left anterior descending

The shunt decreases the risk of electrical or hemodynamic instability, which is a devastating complication in a case without ready access for cardiopulmonary bypass. Final angiographic results after completion of the HCR are presented in Figures 8 and 9. 


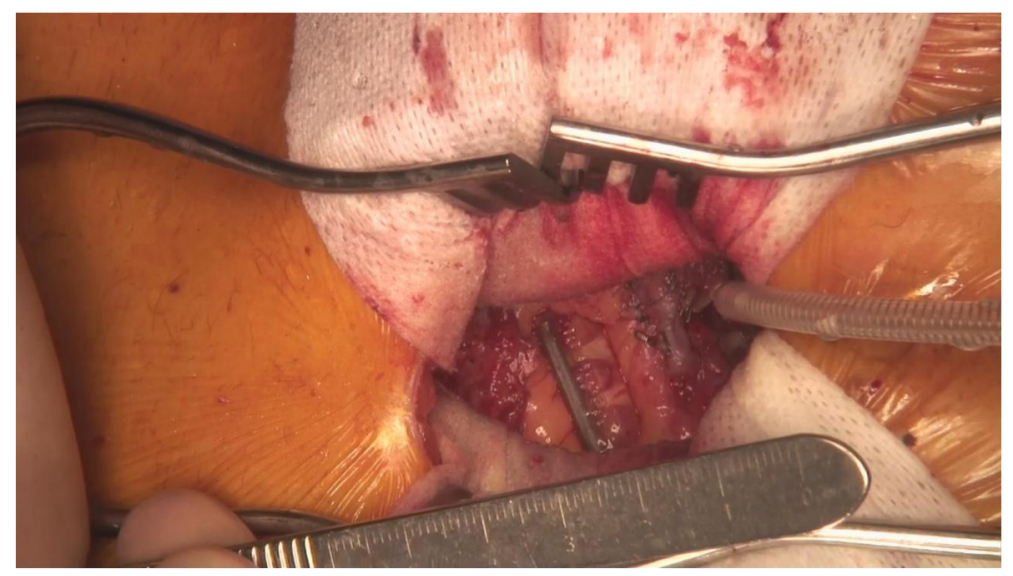

Figure 7. Robotic coronary artery bypass: stabilization and distal anastomosis.

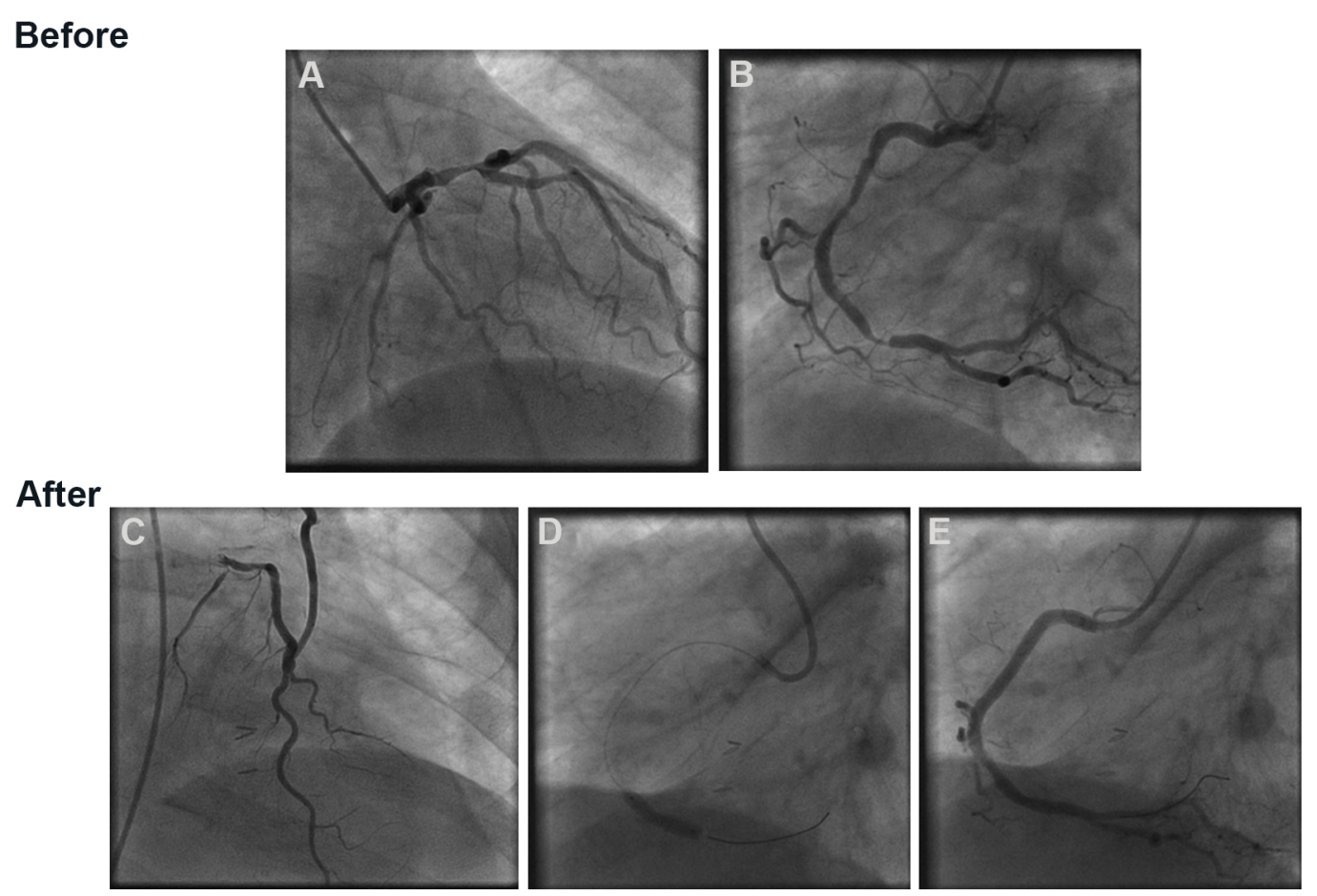

Figure 8. Hybrid coronary revascularization coronary angiogram: robotic left internal mammary artery to left anterior descending and stenting of the proximal circumflex artery.

\section{HCR AND CARDIOPULMONARY BYPASS}

Efforts to move towards a minimally invasive approach have been spearheaded by surgeons who developed expertise in $\mathrm{OPCAB}$, interested in combining the benefits of minimally invasive approaches and OPCAB. Therefore, all of our robotic-assisted cases at Emory are performed off-pump for the LIMA LAD anastomosis, using specially designed, minimally invasive tissue stabilizers for robotic cases. In patients where we suspect we may need a cardiopulmonary bypass, the initial favored approach is a standard median sternotomy. 

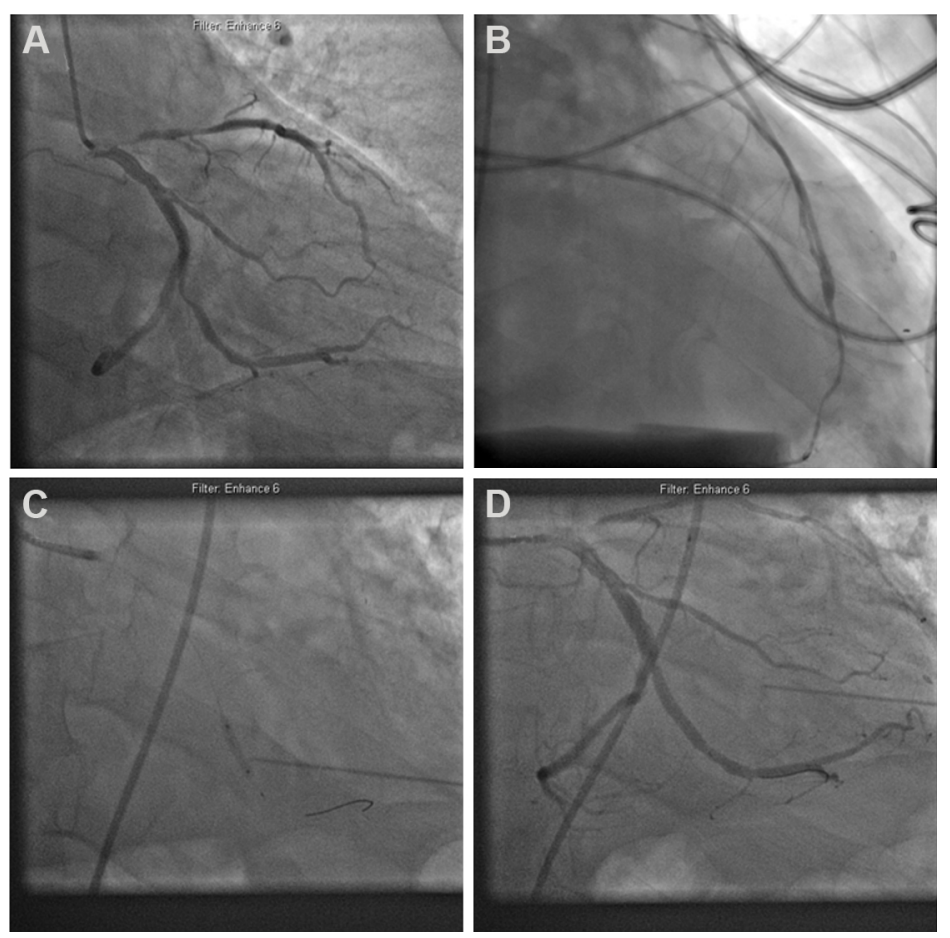

Figure 9. Hybrid coronary revascularization coronary angiogram: robotic left internal mammary artery to left anterior descending and stenting of the distal circumflex artery.

\section{TIMING OF HCR STEPS}

It is important to understand that HCR has two components: minimally invasive or robotic-assisted CABG and PCI. The sequence in which these two steps are performed matters and has clinical, scheduling, and even financial consequences. A comparative summary of the advantages and disadvantages of each sequence is presented in Table 1.

Our preference at Emory is to perform the LIMA-LAD first when possible. In patients with stable coronary disease, this has several advantages, including the ability to perform the surgical procedure without concerns for dual antiplatelet therapy and interrogate the anastomosis angiographically during the subsequent cardiac catheterization. However, for patients that present to the hospital with an acute coronary syndrome, the general rule is to treat the culprit lesion first.

The LIMA LAD anastomosis can be done during the same admission or staged 1-2 months later if the LAD anatomy permits ${ }^{[13,17]}$. The most convenient option for the patient is a concomitant procedure in a hybrid room where the PCI portion of the procedure can be performed immediately after the anastomosis.

The main advantage for the clinician is that graft patency can be confirmed immediately on the operating table, and any technical complications can be addressed while still in the operating room.

However, the logistical challenges of scheduling for both surgeons and interventionalists can make this difficult. 
Table 1. Comparison of the three strategies available to perform the steps of HCR

\begin{tabular}{|c|c|c|}
\hline Minimally invasive CABG first & Stent first & Same setting \\
\hline \multicolumn{3}{|l|}{ Advantages } \\
\hline Less risk of bleeding during the $C A B G$ & $\begin{array}{l}\text { Bails out to conventional surgery if } \mathrm{PCl} \text { unsuccessful without need for second } \\
\text { surgery; helpful option for CTO PCI of non-LAD vessels }\end{array}$ & Convenient for patient \\
\hline $\begin{array}{l}\text { LIMA LAD provides protection during } \\
\text { subsequent } \mathrm{PCI}\end{array}$ & $\begin{array}{l}\text { Allows immediate, expeditious coronary revascularization in patients presenting } \\
\text { with acute coronary syndromes in non-LAD territories with lesions amenable to } \\
\text { PCI }\end{array}$ & Lower total periprocedural length of stay \\
\hline $\begin{array}{l}\text { Able to study the LIMA LAD anastomosis at time } \\
\text { of subsequent PCI }\end{array}$ & & Most financially efficient \\
\hline \multicolumn{3}{|l|}{ Disadvantages } \\
\hline $\begin{array}{l}\text { Incomplete revascularization during the higher } \\
\text { cardiac demands of postoperative recovery }\end{array}$ & No LIMA LAD protection for multivessel PCI & $\begin{array}{l}\text { Difficult coordination of multiple teams: scheduling is inefficient as one team } \\
\text { has to wait for the other to finish and ties up operating room and cath lab } \\
\text { personnel }\end{array}$ \\
\hline Unsuccessful PCI requires a second surgery & $\begin{array}{l}\text { Highest risk of bleeding during CABG due to need for dual antiplatelet therapy } \\
\text { after stent (extended administration) }\end{array}$ & $\begin{array}{l}\text { Slightly higher risk of bleeding due to loading dose of dual antiplatelet agents } \\
\text { for PCI }\end{array}$ \\
\hline Requires two separate procedures & Requires two separate procedures & Longest procedural duration \\
\hline
\end{tabular}

HCR: Hybrid coronary revascularization; CABG: coronary artery bypass; CTO: complete total occlusion; PCI: percutaneous coronary intervention; LAD: left anterior descending; LIMA: left internal mammary artery.

\section{SELECTION OF PATIENTS FOR HCR AND PERFORMING HCR IN SPECIAL CIRCUMSTANCES}

\section{The ideal candidate for HCR}

Experience has taught us that the best-suited patients for HCR are the ones with proximal, focal coronary lesions in fairly large coronary arteries that have a relatively low burden of calcium. Thus, the mid or mid to distal LAD is the prime target for a minimally invasive approach. On the other hand, an intramyocardial LAD is a relative contraindication for HCR because it is very difficult to identify and trace such a vessel through the limited exposure of small thoracotomy attention should be paid to examine the preoperative cardiac catheterization for straight segments of LAD, particularly ones that tend to move inward in systole more than the rest of the LAD, and particularly in the mid LAD.

Body habitus plays a significant role in the success of HCR. In the surgeon's early experience, the ideal patient should be tall, fairly thin, with large pleural cavities and a relatively small heart. A large heart can make harvesting the LIMA very difficult and increases the risk of either LIMA or cardiac injury. Early in our experience, we required two criteria for inclusion: one was a good LAD target vessel for bypass, and the second was a good body habitus for a minimally invasive left thoracotomy approach. We adhered strictly to this protocol for the first 200-300 cases, but currently, all we require is one of the above. [Table 2]. 
Table 2. Relative indications and contraindications of HCR

\begin{tabular}{ll}
\hline Relative indications & Relative contraindications \\
\hline Low-intermediate SYNTAX score & High SYNTAX score \\
Proximal focal coronary lesions & Left thoracotomy, left lung surgery \\
Low burden of calcium in the coronary arteries & Home oxygen requirements \\
Good target vessels (large LAD) & Hemodynamic instability \\
Large pleural cavity & Preoperative need for intraaortic balloon pump \\
Small heart & Obese (particularly morbidly obese) patients \\
Thin, tall body habitus & Suspicion of intramyocardial LAD \\
\hline
\end{tabular}

SYNTAX score: angiographic grading system that evaluates the complexity of lesions in coronary artery disease, ranging from 0 (least complex) to 60 (most complex) and derived from the "SYNergy between percutaneous coronary intervention with TAXUS stent and cardiac surgery" trial. HCR: Hybrid coronary revascularization.

\section{HCR in patients with chronic obstructive pulmonary diseases}

Chronic obstructive pulmonary diseases (COPD) poses an interesting challenge for the performance of HCR. On one hand, increased lung volumes increase left pleural cavity size and improve visualization and ability to harvest the mammary greatly. On the other hand, medium and small airway obstruction can trap air and make visualization difficult. Our preference is to use double-lumen tubes versus bronchial blockers in such patients as this allows better deflation of the left lung. The use of $\mathrm{CO}_{2}$ insufflation for robotic cases can result in respiratory acidosis and hypotension much faster than in patients without COPD; the anticipation of these issues, administration of bronchodilators, and frequent blood gas checks.

With $\mathrm{CO}_{2}$ insufflation, the other option is to use low tidal volume bilateral lung ventilation during the LIMA harvest and deflate the left lung during the anastomosis. Intermittent bilateral lung ventilation can also be used throughout the procedure. In general, we have found that almost all patients who are not on home supplemental oxygen are able to tolerate either single or low-tidal volume bilateral lung ventilation safely.

HCR in patients with chest wall deformities (prior trauma, chest wall radiation, kypho-scoliosis, pectus deformities)

Such patients can pose substantial challenges for minimally invasive CABG because of difficult visualization and possible unpredictable course or complete occlusion of the LIMA in case of prior trauma with rib fractures or radiation. Thus, again, the decision should be made on a case-by-case basis, taking into account the severity of the deformity, its particular location, the likelihood of direct interference with the operation, and most important the experience of the operator with minimally invasive CABG procedures.

\section{HCR for left main disease}

CABG is considered the standard of care for left main disease (LMD). Recently, however, after the results of the EXCEL trial, PCI has been upgraded as an acceptable alternative for LMD treatment. This has opened the possibility for HCR as a solution for LMD. However, performing a "limited intervention" in cases of LMD during the first step of the HCR can have adverse effects during the higher demands of postoperative recovery after a minimally invasive CABG procedure and until the patient can get completely revascularized with PCI. We compared 27 patients who had HCR with 81 contemporary patients treated with off-pump CABG for LMD. In all but one HCR patient, the left main was stented into the circumflex after LIMA LAD anastomosis. Immediate postoperative and medium-term outcomes were similar, except that the need for perioperative blood transfusions was significantly lower in the HCR group than the sternotomy CABG group. There was a trend towards a higher need for repeat revascularization at a median follow-up of 3.2 years, but not statistically significant - 2 patients in the HCR group vs. 1 patient in the CABG group, 
$P=0.9^{[13]}$. Certainly, this study was small but shows that such an approach is feasible.

In general, this option can be considered in patients with either distal left main bifurcation lesion or any lesion in the left main along with a proximal lad lesion. Isolated ostial or body lesions should not be considered for HCR b/c; there will be significant competitive flow with the LIMA b/c; there will no longer be a proximal lesion after PCI of the left main.

\section{HCR for patients with a low ventricular ejection fraction}

Poor contractility makes any cardiac intervention more difficult, and HCR is no exception. The challenge is augmented by the fact that the heart cannot be fully visualized during the CABG part of the operation. Nevertheless, such patients tend to tolerate poorly marginal oxygenation and ventilation that can occur with single lung ventilation. As a general rule, if cardiopulmonary bypass assistance may be needed, our preference is to perform median sternotomy.

\section{HCR in patients with previous left lung surgery or who had previous left thoracotomies}

In our experience, robotic HCR in those circumstances is generally contraindicated. The situation offers the challenge of creating an adequate working space to harvest the LIMA and perform the LIMA to LAD anastomosis because of previous adhesions. Equally challenging can be the fact that the heart can be displaced much further to the left, particularly after left lower lobectomies. It is paramount, particularly in situations of previous left anterolateral thoracotomies, to verify the patency of the LIMA at the time of preoperative cardiac catheterization, as it could have been injured and ligated during the previous surgery. Also, after left lung cancer surgery, it is possible that the patient had radiation to the chest wall on the left, and this can make harvesting the LIMA exceedingly difficult. For all these reasons, the patient might be better served with a conventional CABG and alternative arterial conduits, but these decisions should be made on a case-by-case basis with considerations for the risks and benefits of each approach.

\section{SAFETY OF HCR}

\section{Quality of the LIMA conduit and LIMA LAD anastomosis}

One of the most important questions about HCR concerns exactly that topic: given the different visualization during harvesting as well as limited exposure during the anastomosis, is this truly a comparable end product to the well-established gold standard results of median sternotomy LIMA to LAD operation? A comparison between our early HCR group versus median sternotomy off-pump CABG group indicates that issues with either the LIMA or the LIMA to LAD anastomosis are potentially more prevalent in the HCR group ${ }^{[14]}$. However, these were rarely clinically driven ischemia events in the HCR group. In addition, almost all of the patients in the hybrid group underwent LIMA angiography, and almost none of the patients in the CABG group underwent postoperative angiography. Thus, the comparisons were not standardized, and minor defects early after anastomosis are more likely to be identified.

Our recommendation is to perform completion or postoperative angiography during the surgeon's early experience with robotic-assisted CABG for quality control purposes. This was our model for almost all of the first 3-400 cases. This provides opportunities for refinements in technique, ensures optimal quality outcomes, and ensures that excellent results are achieved with minimally invasive approaches. Our current patency rate approaches $98 \%$ for patients who underwent completion or postoperative catheterization.

One important question is what to do if a mild narrowing or physiologically insignificant defect is detected at or near the distal anastomosis when PCI is performed during the second stage of HCR. There is no compelling literature data about this issue. Certainly, an argument could be made about using invasive 
functional evaluation (fractional flow reserve calculations - iFR/RFR); however, technical issues that can occur in attempts to cross a fresh anastomosis with a wire could be a problem. Our experience, which we are in the process of analyzing for mid and longer-term results, has been that the majority of these should not be intervened on early, particularly if there is TIMI 3 flow distal to the anastomosis. Instead, repeat angiography in 6-8 weeks, and possible iFR/RFR is recommended, and if necessary, intervention can then be performed.

\section{Major adverse cardiac and cerebrovascular events (MACCE - death, myocardial infarction, and stroke)}

In most published series, there are no statistically significant differences in hospital MACCE between the HCR group when compared to our conventional sternotomy patients ${ }^{[14,18-22]}$. However, our current experience suggests a low risk-adjusted mortality rate $<1 \%$ and a stroke rate that is comparable to PCI at approximately $0.5 \%$.

One important finding was that the incidence of perioperative myocardial infarction was not statistically different $(0.7 \% \text { vs. } 0.5 \% \text { in the HCR vs. the conventional group, } P=0.8)^{[14]}$. This alleviates concerns that partial revascularization, either by single vessel CABG or PCI, during the initial part of the procedure would increase the risk of perioperative myocardial infarction due to increased risk of perioperative demand ischemia during the interim period between both procedures ${ }^{[18-22]}$.

In general, if patients present with an acute coronary syndrome secondary to a non-LAD culprit lesion, they should undergo PCI of the non-LAD culprit lesion first. If the LAD lesion is not critical, it can be staged 4-6 weeks later.

If the LAD lesion and the non-LAD lesion(s) are both critical, LIMA LAD grafting should be done first, and PCI should be done postoperatively during the same hospital stay. For non-critical lesions, the procedures can be staged over weeks.

The goals of HCR should be the same as for CABG and multivessel PCI - complete revascularization for all patients.

\section{Risk of bleeding}

An important question about HCR is the risk of bleeding due to the mandatory need for antiplatelet agents in the perioperative period. A pivotal role in the success of HCR is played by the use of DES. Such stents have a long-term patency rate comparable to vein grafts, and second-generation DES are less thrombogenic compared to bare metal stents; however, early thrombosis due to delayed endothelialization can still be an issue. Dual antiplatelet therapy is mandatory after DES, and the risk of stent thrombosis, with associated myocardial infarction or sudden death, doubles for the first generation stents if that therapy is stopped ${ }^{[7,8,23]}$. Even with the second-generation DES, permanent discontinuation of dual antiplatelet therapy before thirty days from stent insertion results in a high risk of stent thrombosis (hazard ratio $=26.8,95 \%$ confidence interval: 8.4-85.4, $P<0.0001$ ); permanent discontinuation after 90 days does not seem to be associated with a higher risk of stent thrombosis ${ }^{[23]}$.

For patients that have undergone a PCI first strategy for HCR, we recommend the continuation of DAPT even for their surgery. Modifications of these recommendations will depend on guideline changes for the duration of DAPT for the latest generation of DES. 
At Emory, we use a staged strategy preferentially, performing the robotic LIMA LAD first, then the PCI, unless the culprit vessel is a non-LAD vessel, in which case PCI is performed first. With this strategy, our blood transfusion requirements have been statistically significantly less in the HCR group than in the sternotomy CABG group $(35.4 \% \text { vs. } 56 \%, P<0.001)^{[14]}$. Similar results have been confirmed by others ${ }^{[17]}$. However, a larger study is necessary to elucidate this issue, as it is possible that performing PCI routinely first, before the portion of the procedure, can increase the risk of bleeding due to the more widespread use of dual antiplatelet therapy. Our current transfusion rate for HCR procedures is approximately $15 \%$ of patients undergoing robotic-assisted CABG, which is significantly less than sternotomy patients - 25\%-30\%.

A word of caution should also be said about the particular type of antiplatelet agents used: most of the studies have been done with the combination of Aspirin and Plavix (Clopidogrel). Newer agents like Brilinta (Ticagrelor) or Effient (Prasugrel) have not been studied extensively in this setting, and it is possible, particularly if PCI is done first and particularly if Effient (which is much more potent at platelet inhibition) is used, that the bleeding complications will be higher in the HCR group.

For patients on these newer generation antiplatelet agents, we usually transition them to clopidogrel if they are sensitive to this agent 7 days before surgery to avoid performing surgery on ticagrelor or prasugrel.

\section{Mistaking a diagonal branch for the LAD}

Mistaking a diagonal branch for the LAD can occur at times, particularly if the LAD is small, intramyocardial or the target LAD lesion is very distal, as mentioned above. If there is no stenosis between the ostium of the diagonal and the LAD, grafting a LIMA to diagonal instead of the LAD has two main drawbacks: the diagonals are much smaller vessels, and the diagonals lack septal perforators, which reduces the vascular bed available for immediate perfusion substantially. Both of these factors can result in decrease patency rates for the LIMA and inadequate long-term flow in the anterior region of the heart.

For these reasons, if the mistake is recognized intraoperatively, we recommend transecting the LIMA as close as possible to the diagonal anastomosis after applying a small clip on the LIMA flush with the diagonal and re-grafting the LAD with the LIMA. If this complication is recognized during the index procedure, it almost always can be addressed during the same setting by dividing the LIMA at the diagonal anastomosis and grafting it onto the LAD.

\section{ADVANTAGES OF HCR: WHAT HCR CAN DO WELL AND WHAT IT DOES ONLY MARGINALLY BETTER THAN CONVENTIONAL CABG}

The main advantages to a hybrid approach are the following:

(1) The major benefit of CABG is still achieved with LIMA LAD grafting;

(2) There is a lower transfusion rate $e^{[14,17]}$ compared to conventional CABG;

(3) Risk of stroke is lower because there is no aortic manipulation and no cardiopulmonary bypass;

(4) The risk of serious wound complications (mediastinitis) is avoided;

(5) Return to normal activity and recovery time are much quicker;

(6) Improved cosmesis.

The main goal with HCR is for patients with proximal LAD disease who may have otherwise been treated with PCI to the LAD can derive the long-lasting benefits associated with LIMA LAD grafting. In addition, most of the patients that undergo robotic-assisted CABG would have otherwise been treated with multivessel PCI, not CABG. 


\section{DEVELOPING A SUCCESSFUL HCR PROGRAM}

The prerequisite for a surgeon to start a successful HCR program is to master the techniques of at least one minimally invasive CABG approach and off-pump coronary bypass. These two goals are difficult to be tackled simultaneously. Off-pump LIMA LAD grafting is best mastered in open sternotomy cases under the careful supervision of a seasoned mentor. However, the pathway for training could be different for a young surgeon just out of training $v s$. an experienced surgeon routinely performing the on-pump, arrested technique. Training in a program experienced with off-pump and beating heart surgeries in its different varieties or joining a group with extensive expertise in such techniques would be the best path forward for the young surgeon. The surgeon will be coached to avoid serious mistakes and gain the expertise and confidence to become a skilled surgeon. These skills can then be translated into a minimally invasive platform.

For the seasoned surgeon with expertise in on-pump arrested CABG, a short period of observation in a busy off-pump program, followed by the transition to performing LIMA to LAD anastomosis on a beating heart, but in a pump assisted fashion, followed by the performance of LIMA to LAD completely off-pump in conjunction with conventional on-pump, arrested technique for the other anastomoses, would lead to expertise during one's own practice.

The harvesting of the mammary artery can be learned as a second step or simultaneously. A minithoracotomy approach harvest is probably the easiest to learn in a self-taught manner after observing cases and watching videos. However, due to the complexities of positioning the robot, training a whole team, and actually learning the technique, robotic cases are a much more ambitious goal and would require either a mini-fellowship or be reserved as a later goal.

First of all, the exposure, be it mini-thoracotomy or a 2-inch, the non-rib spreading incision used for robotic cases, offers a very different view of the heart than a surgeon is used to traditionally. This translates into difficulties in identifying the LAD target.

From the minimally invasive view, the LAD is the furthest vessel to the right; problems can appear, however, when the LAD is buried in epicardial fat or is intramyocardial. Therefore, it is paramount to make sure that the vessel furthest to the right on the anterior aspect of the heart has a general direction tracking to the apex of the heart. This can be verified by direct inspection through a minithoracotomy approach or by camera inspection with the robot and marking the vessel with a marking pen or clip after opening the pericardium robotically prior to performing the small access incision for the LIMA to LAD anastomosis.

\section{CONCLUSION}

In conclusion, HCR is a novel technique that can offer the next level of care for appropriately selected patients in the hands of expert surgeons, combining the benefit of long-term results offered by sternotomy CABG with rapid, short-term recovery and minimal morbidity. Such an approach is feasible if time and energy are invested in the training and logistical development of a collaborative approach.

\section{DECLARATIONS}

\section{Authors' contributions}

Conception, design and editing of the article: Pusca SV, Halkos ME 


\section{Availability of data and materials}

Not applicable.

\section{Financial support and sponsorship}

None.

\section{Conflicts of interest}

Both authors declared that there are no conflicts of interest.

\section{Ethical approval and consent to participate}

Not applicable.

\section{Consent for publication}

Consent for photography is obtained routine for every patient operated at Emory Healthcare Hospitals.

\section{Copyright}

(C) The Author(s) 2021.

\section{REFERENCES}

1. Lytle BW, Blackstone EH, Sabik JF, Houghtaling P, Loop FD, Cosgrove DM. The effect of bilateral internal thoracic artery grafting on survival during 20 postoperative years. Ann Thorac Surg 2004;78:2005-12; discussion 2012-4. DOI PubMed

2. Takagi H, Goto SN, Watanabe T, Mizuno Y, Kawai N, Umemoto T. A meta-analysis of adjusted hazard ratios from 20 observational studies of bilateral versus single internal thoracic artery coronary artery bypass grafting. J Thorac Cardiovasc Surg 2014;148:1282-90. DOI PubMed

3. Voutilainen SM, Järvinen AA, Verkkala KA, et al. Angiographic 20-year follow-up of 61 consecutive patients with internal thoracic artery grafts. Ann Surg 1999;229:154-8. DOI PubMed PMC

4. Tatoulis J, Buxton BF, Fuller JA. Patencies of 2,127 arterial to coronary conduits over 15 years. Ann Thorac Surg 2004;77:93-101. DOI PubMed

5. Serruys PW, Morice MC, Kappetein AP, et al; SYNTAX Investigators. Percutaneous coronary intervention versus coronary-artery bypass grafting for severe coronary artery disease. N Engl J Med 2009;360:961-72. DOI PubMed

6. Leon MB, Mauri L, Popma JJ, et al; ENDEAVOR IV Investigators. A randomized comparison of the Endeavor zotarolimus-eluting stent versus the TAXUS paclitaxel-eluting stent in de novo native coronary lesions 12-month outcomes from the ENDEAVOR IV trial. $J$ Am Coll Cardiol 2010;55:543-54. DOI PubMed

7. Dangas GD, Serruys PW, Kereiakes DJ, et al. Meta-analysis of everolimus-eluting versus paclitaxel-eluting stents in coronary artery disease: final 3-year results of the SPIRIT clinical trials program (Clinical Evaluation of the Xience V Everolimus Eluting Coronary Stent System in the Treatment of Patients With De Novo Native Coronary Artery Lesions). JACC Cardiovasc Interv 2013;6:914-22. DOI PubMed

8. Hess CN, Lopes RD, Gibson CM, et al. Saphenous vein graft failure after coronary artery bypass surgery: insights from PREVENT IV. Circulation 2014;130:1445-51. DOI PubMed PMC

9. Fihn SD, Gardin JM, Abrams J, et al; American College of Cardiology Foundation/American Heart Association Task Force. 2012 $\mathrm{ACCF} / \mathrm{AHA} / \mathrm{ACP} / \mathrm{AATS} / \mathrm{PCNA} / \mathrm{SCAI} / \mathrm{STS}$ guideline for the diagnosis and management of patients with stable ischemic heart disease: a report of the American College of Cardiology Foundation/American Heart Association task force on practice guidelines, and the American College of Physicians, American Association for Thoracic Surgery, Preventive Cardiovascular Nurses Association, Society for Cardiovascular Angiography and Interventions, and Society of Thoracic Surgeons. Circulation 2012;126:e354-471. DOI PubMed

10. Benetti FJ, Ballester C, Sani G, Doonstra P, Grandjean J. Video assisted coronary bypass surgery. J Card Surg 1995;10:620-5. DOI PubMed

11. Vassiliades TA Jr, Reddy VS, Puskas JD, Guyton RA. Long-term results of the endoscopic atraumatic coronary artery bypass. Ann Thorac Surg 2007;83:979-84; discussion 984-5. DOI PubMed

12. Halkos ME, Vassiliades TA, Myung RJ, et al. Sternotomy versus nonsternotomy LIMA-LAD grafting for single-vessel disease. Ann Thorac Surg 2012;94:1469-77. DOI PubMed

13. Halkos ME, Rab ST, Vassiliades TA, et al. Hybrid coronary revascularization versus off-pump coronary artery bypass for the treatment of left main coronary stenosis. Ann Thorac Surg 2011;92:2155-60. DOI PubMed

14. Halkos ME, Vassiliades TA, Douglas JS, et al. Hybrid coronary revascularization versus off-pump coronary artery bypass grafting for the treatment of multivessel coronary artery disease. Ann Thorac Surg 2011;92:1695-701; discussion 1701-2. DOI PubMed

15. Halkos ME, Liberman HA, Devireddy C, et al. Early clinical and angiographic outcomes after robotic-assisted coronary artery bypass surgery. J Thorac Cardiovasc Surg 2014;147:179-85. DOI PubMed

16. Kayatta MO, Halkos ME, Puskas JD. Hybrid coronary revascularization for the treatment of multivessel coronary artery disease. Ann 
Cardiothorac Surg 2018;7:500-5. DOI PubMed PMC

17. Zhou S, Fang Z, Xiong H, et al. Effect of one-stop hybrid coronary revascularization on postoperative renal function and bleeding: a comparison study with off-pump coronary artery bypass grafting surgery. J Thorac Cardiovasc Surg 2014;147:1511-6.e1. DOI PubMed

18. Harskamp RE, Walker PF, Alexander JH, et al. Clinical outcomes of hybrid coronary revascularization versus coronary artery bypass surgery in patients with diabetes mellitus. Am Heart J 2014;168:471-8. DOI PubMed

19. Harskamp RE, Bagai A, Halkos ME, et al. Clinical outcomes after hybrid coronary revascularization versus coronary artery bypass surgery: a meta-analysis of 1,190 patients. Am Heart J 2014;167:585-92. DOI PubMed

20. Harskamp RE, Brennan JM, Xian Y, et al. Practice patterns and clinical outcomes after hybrid coronary revascularization in the United States: an analysis from the society of thoracic surgeons adult cardiac database. Circulation 2014;130:872-9. DOI PubMed

21. Harskamp RE, Vassiliades TA, Mehta RH, et al. Comparative effectiveness of hybrid coronary revascularization vs coronary artery bypass grafting. J Am Coll Surg 2015;221:326-34.e1. DOI PubMed

22. Rosenblum JM, Harskamp RE, Hoedemaker N, et al. Hybrid coronary revascularization versus coronary artery bypass surgery with bilateral or single internal mammary artery grafts. J Thorac Cardiovasc Surg 2016;151:1081-9. DOI PubMed

23. Généreux P, Rutledge DR, Palmerini T, et al. Stent Thrombosis and dual antiplatelet therapy interruption with everolimus-eluting stents: insights from the Xience V Coronary Stent System Trials. Circ Cardiovasc Interv 2015;8:e01362. DOI PubMed 